\title{
Impact of Dengue Fever on Pregnancy and Its Outcome
}

\author{
Suvarna Vishnu Nimkar ${ }^{1}$ \\ ${ }^{1}$ Department of Obstetrics and Gynaecology, Sengupta Hospital and Research Institute, Nagpur, Maharashtra, India.
}

\section{ABSTRACT}

\section{BACKGROUND}

Dengue fever is a vector-borne disease common in the tropics. It is known to have an adverse effect on pregnant women with unfavourable foetal outcomes. However, only a few studies are addressing this problem. Many of the studies had confounding factors such as the simultaneous occurrence of other vector-borne diseases and maternal health conditions. Therefore, the actual impact of dengue fever on pregnancy and pregnancy outcome is not clear. We wanted to study maternal and foetal outcomes in dengue positive pregnant females.

\section{METHODS}

This is a retrospective study done over 6 months in a tertiary care institute. Data regarding demography, obstetric history, biochemical parameters / blood investigation and pregnancy outcomes were collected and analysed.

\section{RESULTS}

Most of the dengue positive women presented with fever and thrombocytopenia in $44 \%$ of cases. Obstetric complications such as preterm labour, abruption and postpartum haemorrhage (PPH) were common. Caesarean section rate was increased and the most common indication was foetal distress. The need for new-born intensive care unit (NICU) admission for new-born and foetal death was also increased. No evidence of congenital anomaly was found in the study.

\section{CONCLUSIONS}

Antenatal complications like preeclampsia, oligohydramnios; intrapartum complications like abruption, DIC, foetal distress and immediate postpartum complications like postpartum haemorrhage, need for blood, platelet transfusion were common in women with a history of dengue fever especially more in the low platelet group. The maternal and foetal outcomes were compromised and both required intensive care. A high index of clinical suspicion is essential in pregnant women presenting with fever and thrombocytopenia. Early diagnosis and management are essential.

\section{KEY WORDS}

Dengue Fever, Dengue in Pregnancy, Complications of Dengue Fever in Pregnancy
Corresponding Author: Dr. Suvarna Vishnu. Nimkar, Department of $O B G$, Sengupta Hospital and Research Institute, Nagpur, Maharashtra, India.

E-mail: suvarnanimkar@gmail.com

DOI: $10.14260 / j e m d s / 2021 / 757$

How to Cite This Article:

Nimkar SV. Impact of dengue fever on pregnancy and its outcome. J Evolution Med Dent Sci 2021;10(44):3742-3746, DOI: $10.14260 /$ jemds/2021/757

Submission 21-07-2021, Peer Review 11-11-2021, Acceptance 17-11-2021, Published 30-11-2021.

Copyright (C) 2021 Suvarna Vishnu Nimkar. This is an open access article distributed under Creative Commons Attribution License [Attribution 4.0 International (CC $B Y 4.0)]$ 


\section{BACKGROUND}

Dengue is one of the most rapidly spreading vector-borne viral diseases in the world. According to the World Health Organization (WHO), approximately $40 \%$ of the world's population (over 2.5 billion people) live in areas with a high risk of contracting dengue infection. ${ }^{1}$ Dengue is caused by virus serotypes of the genus flavivirus, family flaviviridae and the most common vector is Aedes aegypti.

The dengue virus comprises four distinct serotypes (DEN 1-4) but DEN-3 is frequently associated with severe disease. ${ }^{2}$ Infection by one serotype produces lifelong immunity to that specific serotype but only a few months of immunity to the others. ${ }^{3}$ Infection with other kinds of dengue viruses in a person who already had dengue fever in the past is called secondary dengue and is severe. 4 Primary infection is characterized by a slow and low titer antibody response; IgM is the first one to appear. In contrast during a secondary infection, antibody titers rise extremely rapidly. High levels of IgG are detectable even in the acute phase. ${ }^{5}$ We could not differentiate secondary from tertiary or quaternary dengue so some patients classified as having secondary dengue could have had tertiary or quaternary dengue. ${ }^{4}$

As the incidence of dengue in adults increases, the incidence in pregnant women also increases. The majority of dengue patients have a mild self-limiting illness and a few progress to severe disease. ${ }^{6}$ Intravenous rehydration is the treatment of choice, which can reduce the mortality in dengue to less than $1 \%$ of severe cases. ${ }^{2}$ Dengue poses a significant threat to pregnant women.

The clinical severity of the disease has a wide spectrum, and according to the World Health Organization (WHO) dengue classification scheme, it ranges from uncomplicated dengue fever (DF) to dengue haemorrhagic fever (DHF) and devastating dengue shock syndrome (DSS).

\section{Dengue Haemorrhagic Fever (DHF) is Currently Defined by the Following Four WHO Criteria ${ }^{2}$}

- Fever or recent history of fever lasting 2-7 days

- Any haemorrhagic manifestation (petechiae, purpuric lesions, haematemesis, melena, etc.)

- Thrombocytopenia (platelet count of $<100,000 / \mathrm{mm} 3$ )

- Evidence of increased vascular permeability (Ascites, pleural effusion, etc.)

Grade I DHF is mild; the only haemorrhagic manifestations are scattered petechiae and/or positive tourniquet test and Grade II DHF is more severe, with one or more of the overt haemorrhagic manifestations. Grades III and IV represent more severe forms of dengue haemorrhagic fever also known as dengue shock syndrome. Grade III is a mild shock with signs of circulatory failure and grade IV represents profound shock with undetectable pulse \& blood pressure.

Dengue fever happens in three stages: The febrile phase, the Critical phase and the Recovery phase. Rapid NS1 antigen is detected on day 3 of fever and dengue IgM is detected after day 5 of fever. Full blood count as a baseline as well as monitoring the progress of the disease is the most important tool. ${ }^{7}$
Literature search reveals that dengue infection in pregnancy carries the risk of haemorrhage for both the mother and the new-born. In addition, there is a risk of premature birth and foetal death and vertical transmission causing neonatal thrombocytopenia that necessitates platelet transfusions. According to 'The Dengue in pregnancy management protocol 2014', dengue fever does not warrant termination of pregnancy. ${ }^{7}$ There is insufficient data of probable embryopathy to mothers who had dengue fever in the first trimester. ${ }^{7}$ Diagnosis of dengue infection affects management options and decisions of the obstetricians, particularly the mode of delivery due to the potential risk of haemorrhage secondary to thrombocytopenia.

However, there are only a few studies addressing this problem. Thus, this study was conducted to establish the actual impact of dengue fever on pregnancy and the outcome of pregnancy.

\section{Objectives}

- To study antepartum, intrapartum, postpartum complications in pregnant females with dengue fever.

- To assess the maternal and foetal outcomes in dengue positive pregnant females.

\section{METHODS}

The retrospective analysis of all pregnant women with dengue fever was done over 6 months from July 2017 to November 2017 at a tertiary care institute in a metropolitan city in India, irrespective of their gestational age. A total of 22 dengue positive pregnant females were admitted during this period but only 18 were included in the study due to insufficient data required for the study.

\section{Sampling Method}

Cases were selected by convenience sampling method. Dengue positive pregnant women diagnosed using ELISA method by detection of dengue virus-specific NS 1 antigen and/or IgM, IgG antibody were included in the study. The age, gestational age, detailed history, clinical features, complete blood count, liver function test, renal function test, coagulation profiles were noted. Antepartum, intrapartum and postpartum complications were recorded. The mode of delivery was noted. To assess the foetal outcome, NICU admissions, neonatal deaths and babies transfer to mother were recorded. Reasons for NICU admissions were also noted.

\section{Inclusion Criteria}

Pregnant women who delivered during the stated period with confirmed dengue fever in their present antenatal period, irrespective of their gestational age.

\section{Exclusion Criteria}

Women who did not have a report of confirmed dengue fever. 


\section{Statistical Methods}

All the results were plotted in a master chart. Descriptive frequencies, percentages and charts were used, and data analysis was done. Data were analysed and statistics were obtained using IBM SPSS software.

\section{RESULTS}

Cases collected were in the age group of 18-33 years. The mean age at the time of presentation was 24 years \pm 4 years. In the present study, $33.3 \%(6 / 18)$ women were primigravida and $66.7 \%(12 / 18)$ women were multigravida. Most of the women (55.55 \%) were dengue NS1 positive.

\begin{tabular}{|cc|}
\hline Biological Test & Percentage Cases \\
Dengue NS1 positive only & $55.55 \%(10 / 18)$ \\
Dengue IgM positive only & $27.7 \%(5 / 18)$ \\
Dengue NS1+ IgM positive & $11.11 \%(2 / 18)$ \\
Dengue NS1 +IgM + IgG positive & $5.55 \%(1 / 18)$ \\
\hline Table 1. Serological Reporting of Dengue Cases \\
\hline
\end{tabular}

In the present study, associated vector-borne diseases such as malaria were present in $11.1 \%(2 / 18)$ cases and typhoid was present in $5.56 \%(1 / 18)$ cases.

Patients who presented in 1st trimester were $16.7 \%$ $(3 / 18), 2$ nd trimester were $5.6 \%(1 / 18)$ and 3rd trimester were $77.7 \%(14 / 18)$. Most of the dengue positive women presented in the 3rd trimester were in labour at the time of admission. In the present study, platelet count at the time of admission was less than 50,000/mm3 in $16.7 \%$ (3/18) cases, 50,000-1.5 lakh in $27.8 \%(5 / 18)$ cases and > 1.5 lakh in 55.6 $\%(10 / 18)$ cases.

\begin{tabular}{|cccc|}
\hline Presenting & $<\mathbf{5 0 , 0 0 0}$ & $\mathbf{5 0 , 0 0 0 - 1 . 5}$ lakh & $\mathbf{> 1 . 5}$ lakh \\
Symptoms & $\mathbf{( 3 / 1 8 )}$ & $\mathbf{( 5 / 1 8 )}$ & $\mathbf{( 1 0 / 1 8 )}$ \\
Fever & $100 \%(3 / 3)$ & $100 \%(5 / 5)$ & $100 \%(10 / 10)$ \\
Generalized body ache & $\mathrm{Nil}$ & $60 \%(3 / 5)$ & $20 \%(2 / 10)$ \\
Breathlessness & $66.7 \%(2 / 3)$ & $\mathrm{Nil}$ & $10 \%(1 / 10)$ \\
Rash & $33.3 \%(1 / 3)$ & $\mathrm{Nil}$ & $10 \%(1 / 10)$ \\
Black stools & $33.3 \%(1 / 3)$ & $\mathrm{Nil}$ & $\mathrm{Nil}$ \\
Cough \& cold & $\mathrm{Nil}$ & $\mathrm{Nil}$ & $10 \%(1 / 10)$ \\
Vomiting & $\mathrm{Nil}$ & $\mathrm{Nil}$ & $10 \%(1 / 10)$ \\
\hline \multicolumn{4}{c}{ Table 2. Presenting Symptoms } \\
\hline
\end{tabular}

\begin{tabular}{|c|c|c|c|}
\hline Maternal & $<50000$ & \multicolumn{2}{|c|}{$50000-1.5$ lakh $>1.5$ lakl } \\
\hline $\begin{array}{c}\text { Pregnancy-induced hypertension } \\
\text { (PIH) }\end{array}$ & $33.3 \%(1 / 3)$ & Nil & Nil \\
\hline Antepartum haemorrhage (APH) & Nil & Nil & Nil \\
\hline Pleural effusion & $100 \%(3 / 3)$ & $20 \%(1 / 5)$ & $30 \%(3 / 10)$ \\
\hline Hepatomegaly & $33.3 \%(1 / 3)$ & Nil & $10 \%(1 / 10$ \\
\hline \multicolumn{4}{|c|}{ Fetal } \\
\hline Foetal growth restriction (FGR) & $\mathrm{Nil}$ & $20 \%(1 / 5)$ & Nil \\
\hline Oligohydramnios & $33.3 \%(1 / 3)$ & $20 \%(1 / 5)$ & $20 \%(2 / 10)$ \\
\hline \multicolumn{4}{|c|}{ Table 3. Antepartum Complications } \\
\hline
\end{tabular}

\begin{tabular}{|cccc|}
\hline Maternal & $<\mathbf{5 0 0 0 0}$ & $\mathbf{5 0 0 0 0 - 1 . 5}$ lakh & $>\mathbf{1 . 5}$ lakh \\
Abruption & $66.7 \%(2 / 3)$ & $\mathrm{Nil}$ & $\mathrm{Nil}$ \\
$\begin{array}{c}\text { Disseminated intravascular } \\
\text { coagulation (DIC) }\end{array}$ & $33.3 \%(1 / 3)$ & $\mathrm{Nil}$ & $\mathrm{Nil}$ \\
\multicolumn{4}{|c}{ Foetal } \\
Foetal distress & $66.7 \%(2 / 3)$ & $40 \%(2 / 5)$ & $10 \%(1 / 10)$ \\
\hline \multicolumn{4}{|c}{ Table 4. Intrapartum Complications } \\
\hline \multicolumn{4}{|c}{} \\
\hline
\end{tabular}

\begin{tabular}{|ccc|c|}
\hline Maternal & $<\mathbf{5 0 0 0 0}$ & $\begin{array}{c}\mathbf{5 0 0 0 0 - 1 . 5} \\
\text { lakh }\end{array}$ & $>$ 1.5 lakh \\
Post-partum haemorrhage (PPH) & $33.3 \%(1 / 3)$ & $20 \%(1 / 5)$ & $\mathrm{Nil}$ \\
Need for blood transfusion & $33.3 \%(1 / 3)$ & $60 \%(3 / 5)$ & $30 \%(3 / 10)$ \\
Need for platelet transfusion & $66.7 \%(2 / 3)$ & $\mathrm{Nil}$ & $\mathrm{Nil}$ \\
ICU admissions & $66.67 \%(2 / 3)$ & $\mathrm{Nil}$ & $\mathrm{Nil}$ \\
\hline \multicolumn{4}{|c|}{ Table 5. Immediate Postpartum Complications } \\
\hline \multicolumn{4}{|c}{} \\
\hline
\end{tabular}

The outcome of pregnancy was measured in terms of caesarean section, vaginal delivery and spontaneous abortion. In the present study, $50 \%(9 / 18)$ patients had a caesarean section, $38.9 \%(7 / 18)$ had a vaginal delivery and $11.1 \%(2 / 18)$ had a spontaneous abortion.

In the present study, $62.5 \%(10 / 16)$ of babies born to dengue positive mothers required admission to the neonatal care unit due to various reasons; prematurity and low birth weight were common amongst them. Amongst the neonates admitted to intensive care, 2 deaths $12.5 \%(2 / 16)$ were reported. $37.5 \%(6 / 16)$ of foetuses were healthy, admitted with mothers.

\begin{tabular}{|cc|}
\hline Reasons for NICU Admission & Percentage Cases \\
Low birth weight & $30(3 / 10)$ \\
Asphyxia & $20(2 / 10)$ \\
Meconium aspiration syndrome & $10(1 / 10)$ \\
Hypoxic-ischemic encephalopathy & $10(1 / 10)$ \\
Pulmonary haemorrhage & $10(1 / 10)$ \\
Bruising & $10(1 / 10)$ \\
Neonatal jaundice & $10(1 / 10)$ \\
\hline Table 6. Reasons for NICU Admission \\
\hline
\end{tabular}

\section{DISCUSSION}

The outbreak of dengue is common during the monsoon and post-monsoon season in India.8,9 Fever with thrombocytopenia during pregnancy causes panic among the practising obstetricians. The initial symptoms can be ambiguous, self-limited and vary from myalgia, malaise to intense headache and arthralgia. The physiological changes during pregnancy may mislead the physician's attention. Physiological haemodilution in pregnancy can mask the haemoconcentration in DHF. Elevated liver enzymes with a fall in platelet count and bleeding tendencies can mislead the diagnosis to HELLP syndrome.10 Dengue in pregnancy requires early diagnosis and treatment and it should be considered as a differential diagnosis of fever in mothers during epidemics. ${ }^{11}$

Dengue may be due to primary or secondary infection. Primary infection is defined as an IgM negative/IgG negative; or IgM-positive/IgG-negative on the blood sample drawn within 3 days of symptom onset. Secondary infection is defined as an IgM-negative/IgG-positive or an IgM-positive/ IgG-positive result on the blood sample drawn within 3 days of symptom onset. Days of illness were calculated considering the day of onset of symptoms (like fever, body aches, vomiting etc.) as the first day. ${ }^{4}$ In the present study, most of the patients had primary dengue infection.

The present study had maximum third-trimester cases with most of them presenting in labour and at the time of delivery. Kariyawasam and Senanayake showed in their study that 3 of 15 females presented in the second trimester of pregnancy, maximum i.e., 12 in their third trimester and none in first trimester. ${ }^{3}$

Symptoms of infected women vary among the reports published in the literature. In general, the most common symptoms include fever, myalgia and arthralgia. ${ }^{12}$ In our study, fever was present in all dengue positive patients.

Patients with low platelet count $(<50,000 / \mathrm{mm} 3)$ presented with bleeding tendencies and evidence of plasma leak on the scan. Fluid leakage (elevated haematocrit, pleural 
effusion or ascites) and haemorrhagic manifestations are characteristic features of DHF. The physiological haemodilution of a normal pregnancy can mask the classical criteria of haemoconcentration in DHF. ${ }^{13}$ Routine ultrasound examination for free fluid in abdominal or thoracic cavities may be supplementary and practical in pregnant women. ${ }^{3}$ In the present study, only $16.7 \%$ (3/18) patients had platelet count $<50,000 / \mathrm{mm} 3$ and all had pleural effusion on scan report.

The antepartum, intrapartum and postpartum periods are more complicated when associated with thrombocytopenia. In the present study, $44 \%(8 / 18)$ patients presented with thrombocytopenia (< 1 lakh) out of which $25 \%(2 / 8)$ required platelet transfusion. Similar results were found in a study by S.Kariyawasam et al. in 2010 (46.6\%). ${ }^{3}$

Dengue virus infection in pregnancy increases premature birth risk, especially if the infection occurs in the last trimester of pregnancy. In the present study, $27.8 \%(5 / 18)$ patients had preterm labour and delivery. Data from other authors also showed an increase in the rate of prematurity. Carles et al. in their review of 38 cases in French Guiana indicated a significant increase in prematurity and foetal death. The main obstetric consequences of infection were an increase in premature births (19.6\%) and an increased risk of preterm labour ( $41 \%$ ) cases in the study by Basurko C et al. ${ }^{14}$

Caesarean section rate was found to be more than vaginal delivery in the present study. The most common indication for caesarean section was foetal distress in 3 out of 9 cases $(33.33 \%)$. The overall rate of foetal distress in the third trimester was $27.77 \%$ (5/18). Basurko $C$ et al. also observed foetal distress in $7.5 \%$ of patients whereas Garg R et al. noted a higher rate of $16 \%$ in their study conducted at Agra. ${ }^{14}$ The gestational age at presentation of dengue fever also appeared to be significant. Early-onset and late-onset in pregnancy appeared to have a bad prognosis. In the present study, 11.1 $\%(2 / 18)$ cases had a spontaneous abortion who presented early in pregnancy. Two late miscarriages at 17 and 19 weeks of gestation were observed in the study by Carles et al. ${ }^{15}$

In the present study, $33 \%(1 / 3)$ patients with platelet count $<50,000 / \mathrm{mm} 3 \& 5.5 \%(1 / 18)$ overall had post-partum haemorrhage which was medically managed. Basurko $\mathrm{C}$ et al. also reported postpartum haemorrhage in $9.8 \%$ of cases, one of which had DIC. ${ }^{6}$ In our study, $33 \%(1 / 3)$ patients in low platelet count group $(<50,000 / \mathrm{mm} 3) \& 5.5 \%(1 / 18)$ overall had coagulation failure and DIC.

Haemorrhagic complications like post-partum haemorrhage (PPH) and abruption have been described in studies. We also had 2 cases of abruptio placenta (66\%) in patients with low platelet group $(<50,000 / \mathrm{mm} 3) \& 11 \%$ $(2 / 18)$ overall in the study.

Although cases with disseminated intravascular coagulation, pre-eclampsia, abruption, postpartum haemorrhage and ICU admissions were noted in this study, there was no case of maternal death. Ismail et al. in a recent review reported 3 maternal deaths out of 16 cases (2.6\%) and also noted a $50 \%$ prematurity rate. 16

The mean birth weight of babies born to mothers with dengue was $2.6 \mathrm{~kg}$. Birth weights less than $2.5 \mathrm{~kg}$ were observed in almost $50 \%$ of cases, with a minimum of $1.2 \mathrm{~kg}$. Among these, $27.8 \%$ were preterm and appeared to be having weight adequate for gestational age. Although the low birth weight in the term babies was most likely secondary to pregnancy-induced hypertension in their mothers. The exact cause was not defined. Other studies have also shown similar outcome. ${ }^{14}$

In the present study, $62.5 \%(10 / 16)$ babies required admission to neonatal care unit and the reasons for the same are stated in Table 6. Low birth weight was the most common reason amongst them.

No evidence of any congenital anomaly was found in the present study. Sharma et al. reported an increase in the incidence of foetal neural tube malformation in women who had dengue in the first quarter of pregnancy, but such an association has been demonstrated following other febrile illnesses, due to pyrexia rather than to any teratogenic effect of the virus perse. ${ }^{17}$

\section{CONCLUSIONS}

Both maternal and foetal outcomes were compromised and both required intensive care and monitoring. Antenatal complications like preeclampsia, oligohydramnios; intrapartum complications like abruption, DIC, foetal distress and immediate postpartum complications like postpartum haemorrhage, need for blood, platelet transfusion were common in women with a history of dengue fever especially more in the low platelet group.

Early detection and immediate management can minimize the risk to both mothers as well as to the foetus. Patients with dengue fever especially with low platelet count require frequent follow up antenatally and close monitoring during labour, delivery and the immediate postpartum period. In the absence of associated feto-maternal complications, infection by itself does not appear to be an indication for obstetric interference.

\section{Clinical Implications}

This study will help obstetricians to formulate the management protocols of dengue fever in pregnant females. They can anticipate trimester wise complications in a pregnant lady. Similarly, the need for intensive care and monitoring for thrombocytopenic patients is proven in the study as complications are directly proportional to the severity of thrombocytopenia in pregnant women. Further, preparedness for emergency operation theatre and neonatal care unit is necessary while managing severe cases of dengue fever.

\section{Limitations}

The limitation of this study is the small number of patients. We could not exclude other risk factors of PPH in patients with dengue fever, although the relationship between low platelet count and PPH seems reasonable. Women with a history of dengue fever irrespective of gestational age were included in the study. Thus, further studies are mandatory, as evidence-based data especially in pregnant women, is sparse.

Data sharing statement provided by the authors is available with the full text of this article at jemds.com.

Financial or other competing interests: None. 
Disclosure forms provided by the authors are available with the full text of this article at jemds.com.

\section{REFERENCES}

[1] Mulik V, Dad N, Buhmaid S. Dengue in pregnancy: review article. Eur J Obstet Gynecol Reprod Biol 2021;(2):20510.

[2] World Health Organization. Dengue guidelines for diagnosis, treatment, prevention and control: new edn. World Health Organization 2009.

[3] Kariyawasam S, Senanayake H. Dengue infections during pregnancy: case series from a tertiary care hospital in Sri Lanka. J Infect Dev Ctries 2010;4(11):767-75.

[4] Changal KH, Raina AH, Raina A, et al. Differentiating secondary from primary dengue using IgG to IgM ratio in early dengue: an observational hospital based clinicoserological study from North India. BMC Infect Dis 2016;16(1):715.

[5] Schilling S, Ludolfs D, van An L, et al. Laboratory diagnosis of primary and secondary dengue infection. J Clin Virol 2004;31(3):179-84.

[6] Chitra TV, Panicker S. Maternal and fetal outcome of dengue fever in pregnancy. J Vector Borne Dis 2011;48(4):210-3.

[7] Guideline developmental group meeting. Dengue in pregnancy: management protocols. Dengue Hemorrhagic Fever 2014:1-11.

[8] Gupta E, Dar L, Narang P, et al. Serodiagnosis of dengue during an outbreak at tertiary care hospital in Delhi. Indian J Med Res 2005;121(1):36-8.
[9] Gunasekaran P, Kaveri K, Mohana S, et al. Dengue disease status in Chennai (2006-2008): a retrospective analysis. Indian J Med Res 2011;133(3):322-5.

[10] Veena L. Dengue infection in pregnancy and outcome in a tertiary care centre. Int J Reprod Contracept Obstet Gynecol 2018;7(12):5051-5.

[11] Gehlot H, Yadav OP, Sharma S, et al. A study of dengue fever in pregnancy and its maternal and fetal prognosis. Int J Reprod Contracept Obstet Gynecol 2017;6(8):34147.

[12] Malavige GN, Velathanthiri VGNS, Wijewickrama ES, et al Patterns of disease among adults hospitalized with dengue infections. QJM 2006;99(5):299-305.

[13] Waduge R, Malavige GN, Pradeepan M, et al. Dengue infections during pregnancy: a case series from Sri Lanka and review of the literature. J Clin Virol 2006;37(1):2733.

[14] Kanakalatha DH, Radha S, Nambisan B. Maternal and fetal outcome of dengue fever during pregnancy. 2016;5(11):3959-64.

[15] Basurko C, Carles G, Youssef M, et al. Maternal and foetal consequences of dengue fever during pregnancy. Eur J Obstet Gynecol Reprod Biol 2009;147(1):29-32.

[16] Sharma JB, Gulati N. Potential relationship between dengue fever and neural tube defects in a northern district of India. Int J Gynaecol Obstet 1992;39(4):291-5.

[17] Moretti ME, Bar-Oz B, Fried S, et al. Maternal hyperthermia and the risk for neural tube defects in offspring: systematic review and meta-analysis. Epidemiol 2005;16(2):216-9. 